\title{
Ansiedade em estudantes de medicina no Brasil: uma revisão sistemática
}

\author{
Anxiety in medical students in Brazil: a systematic \\ review
}

DOI: $10.54022 /$ shsv1n1-001

Recebimento dos originais: 06/01/2020

Aceitação para publicação: 31/01/2020

\author{
Jéssica Abreu Silva \\ Maria Adriely Cunha Lima \\ Yasmim Laila Fragoso Cestari \\ Halley Ferraro Oliveira
}

\begin{abstract}
RESUMO
Introdução: O curso de Medicina apresenta alguns fatores estressores, como alta cobrança de resultados, grande carga horária, pressão de professores e familiares além da intensa dedicação aos estudos e contato com sofrimento alheio, que podem desencadear transtornos psiquiátricos. Além disso, por si só o ingresso na universidade é um período de grandes impactos na saúde mental e na vida social desses estudantes. Consequentemente, o aluno desenvolve uma elevada expectativa de conquistas, que pode resultar em estresse e, como consequência, desencadear um quadro de ansiedade. Objetivo: $O$ presente resumo tem como objetivo investigar a ocorrência dos sintomas de ansiedade em estudantes de Medicina. Método: Este estudo é uma revisão sistemática que visa analisar, principalmente, a ansiedade em estudantes de medicina do Brasil. Para isso, foi realizado uma pesquisa através das bases de dados PubMed $\AA$, Biblioteca Virtual em Saúde (BVS) e Scientific Electronic Library Online (SciELO). Após isso, foram definidos como filtros: ensaio clínico, estudo de coorte, ensaio clínico controlado, estudo observacional de corte, transversal, caso-controle e experimental, além de artigos publicados entre 2016 e 2021 e descritores presentes no título. Resultado: A partir desse estudo, foi observado que, principalmente no sexo feminino, os índices da prevalência de ansiedade em estudantes de medicina, têm se apresentam de forma crescente. Outros achados foram de que no total, $28 \%$ dos estudantes de medicina apresentaram ansiedade, sendo que $50 \%$ dos estudos relataram que o sexo feminino estava relacionado com maiores índices de ansiedade, sendo considerado, por alguns, como um fator de risco. Outros fatores associados a isso foi realização de terapia psicológica ou psiquiátrica, distúrbios no sono, uso de drogas e outros. Conclusão: Este estudo alerta sobre os estudantes de medicina possuírem uma tendência maior a desenvolver sintomas de ansiedade ao decorrer de sua formação profissional, pois lidam com um curso que exige muito tanto física quanto emocionalmente, principalmente por trabalhar
\end{abstract}


com as dores e os sentimentos de uma pessoa. Sendo assim, torna-se relevante proporcionar esclarecimentos sobre essa temática na intenção de reafirmar a importância de oferecer cuidados a esses estudantes no decorrer da sua formação.

Palavras-chaves: Ansiedade, Estudantes de medicina, Esgotamento Psicológico, Esforço Físico.

\section{ABSTRACT}

Introduction: The medical course has some stressors, such as high demand for results, large workload, pressure from teachers and family members, in addition to intense dedication to studies and contact with the suffering of others, which can trigger psychiatric disorders. Furthermore, admission to university in itself is a period of great impact on the mental health and social life of these students. Consequently, the student develops a high expectation of achievements, which can result in stress and, as a consequence, trigger anxiety. Objective: This abstract aims to investigate the occurrence of anxiety symptoms in medical students. Method: This study is a systematic review that aims to analyze, mainly, anxiety among medical students in Brazil. For this, a search was carried out through the PubMed $\AA$, Virtual Health Library (BVS) and Scientific Electronic Library Online (SciELO) databases. After that, the following were defined as filters: clinical trial, cohort study, controlled clinical trial, observational, cross-sectional, case-control and experimental study, in addition to articles published between 2016 and 2021 and descriptors present in the title. Result: From this study, it was observed that, especially among females, the prevalence rates of anxiety in medical students are increasing. Other findings were that, in total, $28 \%$ of medical students had anxiety, and $50 \%$ of the studies reported that females were associated with higher levels of anxiety, being considered, by some, as a risk factor. Other factors associated with this were psychological or psychiatric therapy, sleep disorders, drug use and others. Conclusion: This study warns that medical students have a greater tendency to develop symptoms of anxiety during their professional training, as they deal with a course that demands a lot both physically and emotionally, especially for working with the pain and feelings of a people. Therefore, it is relevant to provide clarification on this topic in order to reaffirm the importance of promoting greater care for these students throughout their training.

Keywords: anxiety, medical students, psychological distress, physical exertion.

\section{INTRODUÇÃO}

A palavra "ansiedade" deriva do termo latim "anxietas", o qual significa "angustia, aflição, pertubação causada pela incerteza", ou seja, ela traz a tona às sensações referidas por pessoas que experimentaram esse sentimento ${ }^{1}$. A ansiedade é algo natural do ser humano e todos no mundo alguma vez em vida experimentará esse sentimento, o qual pode aparecer em variadas situações adversas como ameaças, separações e perdas. Essa emoção passa a ser 
patológica quando as suas respostas são desproporcionais e até excessivo a uma situação ansiogênica real ou imaginária, ou até mesmo, o indivíduo se prepara para reagir ao risco mesmo quando não há um risco, fazendo dessa situação um comportamento padrão, e não eventual, além de afetar a qualidade de vida. A causa dessa exacerbação na resposta do sentimento normal ainda não é muito bem conhecida e dessa maneira necessita-se de estudos para melhor avaliar o assunto ${ }^{2,3}$.

Compreender o que significa ansiedade pode se dar sob vários enfoques, sendo que ela pode ser definida como estado emocional que participa das experiências humanas e contém componentes psicológicos e fisiológicos, sendo propulsora do desempenho ${ }^{4}$. Apesar da ansiedade se manifestar de formas diferentes nas pessoas, todo mundo irá experimentá-la em algum momento, sendo que ela poderá se reproduzir como uma sensação difusa, vaga de apreensão ou desagradável, podendo vir acompanhada de outros sintomas como cefaleia, palpitação, aperto no peito, leve mal-estar epigástrico e inquietação ${ }^{2}$. A ansiedade, por ter uma gama de sintomas, se manifesta de maneira diferente entre as pessoas. Tais sintomas podem ser somáticos (dispneia, taquicardia, dores musculares, tremor, outros) e psíquicos (nervosismo, apreensão, insegurança, dificuldade de concentração, outros) ${ }^{5}$.

A faculdade de medicina é um estressor que reduz o desempenho acadêmico, a saúde e o bem-estar psicológico do estudante, sendo que até $50 \%$ desses indivíduos podem ter algum transtorno emocional ${ }^{6}$. Desde 0 início da graduação já ocorre mudanças nos hábitos representando um momento em que há ampliação de conhecimentos, expectativas e experiências e, consequentemente, já é necessário um acompanhamento da saúde mental dos estudantes, visto que muitas vezes eles não estão preparados psicologicamente para responsabilidade e cobrança, medo do fracasso, imposição do mercado de trabalho e outros fatores presente no curso que desgastam o biopsicossocial do acadêmico 7,8. Majoritariamente, os graduandos de medicina adoecem mais quando comparados com a população em geral, sendo que são mais vuneráveis ao estresse e as morbidades associadas a essa patologia ${ }^{6}$.

A ansiedade é uma doença debilitante que tem aumentado sua incidência nos estudantes de medicina, consequentemente, há a necessidade de uma 
atenção maior voltada para esse grupo, visto que isso pode interferir negativamente no desempenho acadêmico, na evasão da faculdade e no desenvolvimento profissional ${ }^{8}$. Sabe-se que diante do estresse, um indivíduo pode ter diferente respostas psicológicas, sendo que as mais comuns são as ansiosas e as depressivas ${ }^{9}$. Referente a tais efeitos sobre o humor, a ênfase neste estudo, se dará, principalmente sobre a ansiedade. Diante do exposto, o presente estudo visa identificar a prevalência dos sintomas associados a ansiedade entre estudantes de Medicina no Brasil, tornando relevante proporcionar esclarecimentos sobre essa temática na intenção de reafirmar a importância de oferecer cuidados a esses estudantes no decorrer da sua formação.

\section{MÉTODO}

Este estudo é uma revisão sistemática que visa analisar, principalmente, a ansiedade em estudantes de medicina do Brasil. Para isso, foi realizado uma pesquisa através das bases de dados PubMed $\AA$, Biblioteca Virtual em Saúde (BVS) e Scientific Electronic Library Online (SciELO) por meio dos descritores definidos "anxiety" (D1), "medical" (D2) e "students" (D3), além do uso do operador Booleano "AND". Sendo assim, foi utilizado a associação "D1" AND "D2" AND "D3", de modo que fosse ampliado o número de artigos encontrados.

Após isso, foram definidos como filtros: ensaio clínico, estudo de coorte, ensaio clínico controlado, estudo observacional de corte, transversal, caso-controle e experimental, além de artigos publicados entre 2016 e 2021 e descritores presentes no título. Além desses filtros, foi definido como critérios de inclusão estudos com a amostra brasileira e o texto disponível na íntegra. Ademais, não houve restrição quanto ao idioma, de forma que não houvesse limitações nos estudos encontrados. No que diz respeito aos critérios de exclusão, foram excluídos artigos que não dissertavam sobre o tema ou não abrangiam o objetivo proposto.

Inicialmente, foram encontrados 4.010 artigos, após aplicar os critérios de inclusão, restaram 232. Após isso, foi realizado a leitura dos títulos e dos resumos para excluir os estudos que não discorriam sobre a temática e as duplicatas, selecionando apenas 23 estudos para leitura integral. Desses, 21 foram 
escolhidos para compor a base de dados dessa revisão (figura 1). É válido salientar que todo processo de seleção dos artigos foi realizado de forma independente por dois revisores.

Figura 1. Diagrama de fluxo PRISMA do estudo.

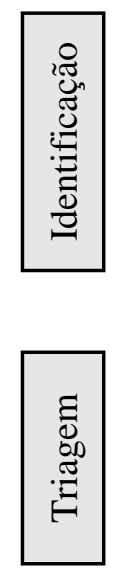

Estudos identificados nas bases de dados $(\mathrm{N}=4.010)$
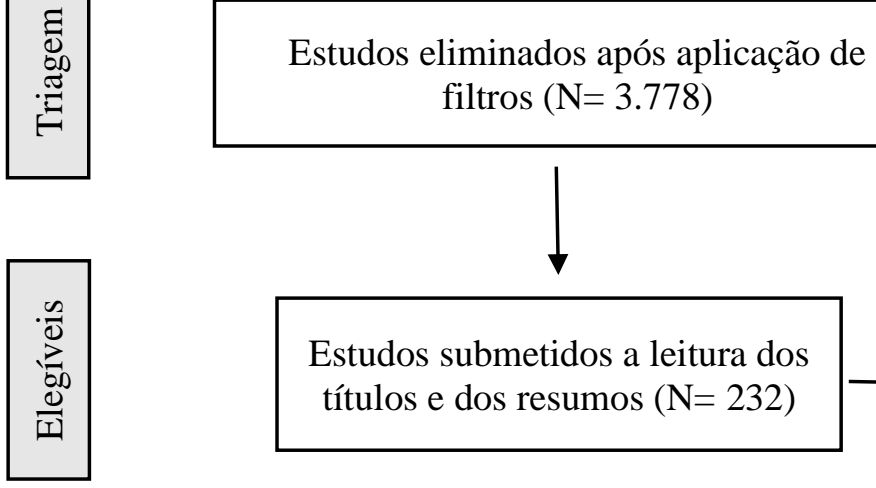
filtros $(\mathrm{N}=3.778)$
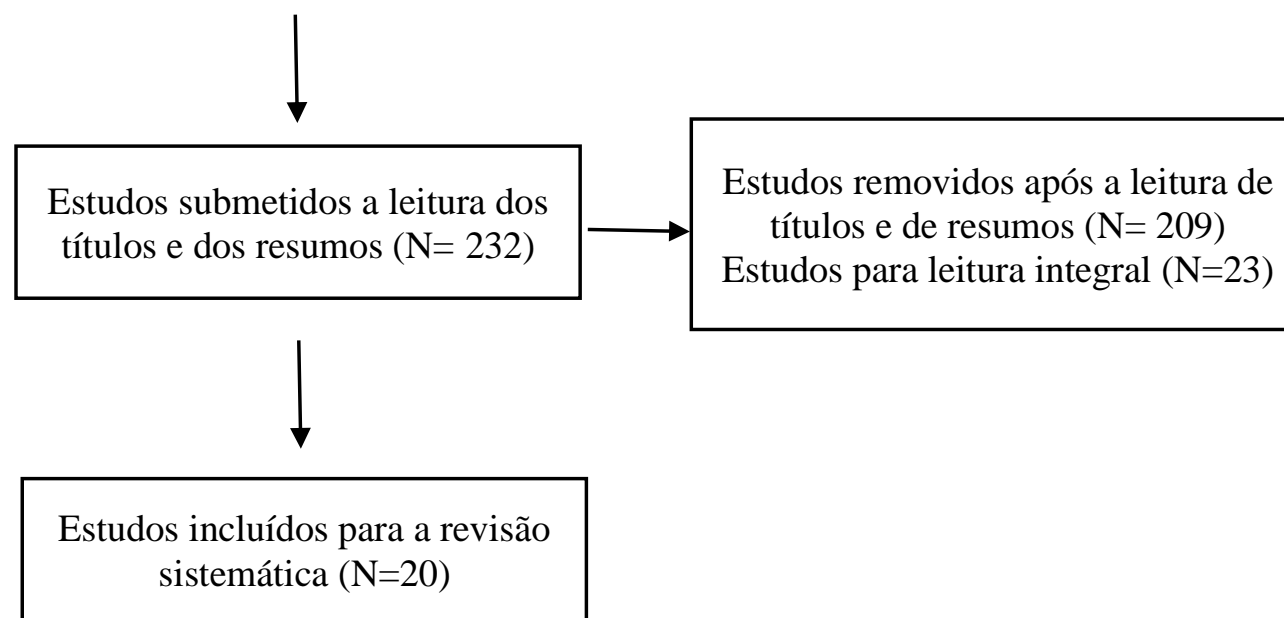

Fonte: dados da pesquisa, 2021.

A seleção dos artigos executada por dois revisores, ML e YC, de forma independente, de modo que fosse possível aplicar o índice Kappa. Sendo que, em caso de ausência de concordância, por exemplo, seria eleito outro revisor (HF), o qual escolheria um artigo no ponto de intersecção dos dois revisores anteriores, a fim de que fosse removido a discrepância inicial (Tabela 2).

Ao aplicar o índice Kappa, foi obtido um valor de 0.8831, o qual segundo Landis e Koch $^{10}$ é interpretado como concordância quase perfeita, sendo possível prosseguir com a revisão sistemática (Tabela 2). 
Tabela 2. Índice de contingência e Índice Kappa.

\begin{tabular}{|c|c|c|c|c|c|}
\hline & & & & \\
\hline & & \multicolumn{4}{|c|}{ Revisor YC } \\
\hline & & Cat. 1- Sim & \multicolumn{2}{|c|}{ Cat. 2- Não } & Total \\
\hline \multirow{3}{*}{ Revisor ML } & Cat. 1- Sim & 19 & 1 & & 20 \\
\hline & Cat. 2- Não & 4 & 3.986 & & 3.990 \\
\hline & Total & 23 & 3.987 & & 4.010 \\
\hline \multicolumn{2}{|c|}{ Índices } & \multicolumn{2}{|c|}{ Categoria $1^{*}$} & \multicolumn{2}{|c|}{ Categoria $2^{\star}$} \\
\hline \multicolumn{2}{|c|}{ Kappa da categoria } & \multicolumn{2}{|l|}{0.8831} & \multicolumn{2}{|c|}{0.8831} \\
\hline \multicolumn{2}{|c|}{ Erro padrão do Kappa da categoria } & \multicolumn{2}{|l|}{0.0522} & \multicolumn{2}{|c|}{0.0519} \\
\hline \multicolumn{2}{|c|}{$\begin{array}{l}\text { Intervalo de } 95 \% \text { de confiança do } \\
\text { Kappa da categoria }\end{array}$} & \multicolumn{2}{|l|}{$\begin{array}{l}\text { Sup: } 0.7807 \\
\text { Inf: } 0.9855\end{array}$} & \multicolumn{2}{|c|}{$\begin{array}{l}\text { Sup: } 0.7814 \\
\text { Inf: } 0.9848\end{array}$} \\
\hline
\end{tabular}

${ }^{*}$ Artigos incluídos no estudo **Artigos excluídos do estudo

Fonte: dados da pesquisa, 2021.

\section{RESULTADO}

No quadro 1 é possível analisar que 45\% $(n=9)$ dos artigos selecionados foram publicados em uma mesma revista. No que diz respeito aos métodos utilizados para avaliar a ansiedade nos estudantes de medicina, o mais prevalente foi o Inventário de Ansiedade Beck (BAI), utilizado em 30\% ( $n=6)$ dos estudos, seguido pela Escala de Ansiedade e Depressão Hospitalar (HADS) e pela Escala de Depressão, Ansiedade e Estresse (DASS-21), em 15\% $(n=3)$ dos artigos.

Quadro 1. Características dos estudos selecionados para revisão.

\begin{tabular}{|c|c|c|c|}
\hline Artigo & Revista & Tipo de estudo & $\begin{array}{c}\text { Escala } \\
\text { aplicada }\end{array}$ \\
\hline Bassols AMS et al ${ }^{11}$ & Revista Brasileira de Psicoterapia & $\begin{array}{l}\text { Observacional } \\
\text { transversal }\end{array}$ & DSQ-40 \\
\hline Bermudez MB et al ${ }^{12}$ & Revista Brasileira de Psiquiatria & Experimental aberto & OASIS \\
\hline Borges GBM et $a l^{13}$ & $\begin{array}{l}\text { Trends in psychiatry and } \\
\text { psychotherapy }\end{array}$ & $\begin{array}{l}\text { Observacional } \\
\text { transversal }\end{array}$ & HADS \\
\hline Costa DS et al ${ }^{14}$ & $\begin{array}{l}\text { Revista Brasileira de Educação } \\
\text { Médica }\end{array}$ & Transversal quantitativo & BAI \\
\hline Damiano RF et al ${ }^{15}$ & Revista Brasileira de Psiquiatria & Transversal exploratório & DASS-21 \\
\hline Del-Bem CM et al ${ }^{16}$ & São Paulo Medical Journal & Coorte & $\mathrm{BAI}$ \\
\hline $\begin{array}{l}\text { Gonçalves JRL et } \\
\text { a/17 }\end{array}$ & $\begin{array}{l}\text { Revista Brasileira de Educação } \\
\text { Médica }\end{array}$ & Transversal quantitativo & HADS \\
\hline Lucchetti G et al ${ }^{18}$ & Academic Psychiatry & $\begin{array}{l}\text { Transversal } \\
\text { transcultural }\end{array}$ & DASS 21 \\
\hline Machado L et al ${ }^{19}$ & $\begin{array}{l}\text { Trends in psychiatry and } \\
\text { psychotherapy }\end{array}$ & Transversal & PSWQ \\
\hline
\end{tabular}




\begin{tabular}{|c|c|c|c|}
\hline Maia JMC et $a^{20}$ & International Review of Psychiatry & Observacional & $\mathrm{gHQ}-12$ \\
\hline Mayer FB et $a l^{21}$ & BMC Medical Education & Coorte multicêntrico & STAI \\
\hline $\begin{array}{l}\text { Moromizato MS et } \\
a^{2}\end{array}$ & $\begin{array}{l}\text { Revista Brasileira de Educação } \\
\text { Médica }\end{array}$ & Transversal quantitativo & BAI \\
\hline Motta ICM et aR3 & $\begin{array}{l}\text { Revista Brasileira de Educação } \\
\text { Médica }\end{array}$ & Quali-quantitativo & MINI \\
\hline Moutinho IVD et al 24 & Psychiatry Research & Observacional & DASS-21 \\
\hline Nogueira EG et al ${ }^{25}$ & $\begin{array}{l}\text { Revista Brasileira de Educação } \\
\text { Médica }\end{array}$ & Transversal quantitativo & BAI \\
\hline Regis JMO et al ${ }^{26}$ & Jornal Brasileiro de Psiquiatria & Transversal & SPIN \\
\hline Ribeiro CF et al ${ }^{27}$ & $\begin{array}{l}\text { Revista Brasileira de Educação } \\
\text { Médica }\end{array}$ & Transversal & HADS \\
\hline $\begin{array}{l}\text { Rodrigues MDS et al } \\
28\end{array}$ & $\begin{array}{l}\text { Revista Brasileira de Educação } \\
\text { Médica }\end{array}$ & Transversal & LSAS-SR e BAI \\
\hline $\begin{array}{l}\text { Sacramento BO et al } \\
29\end{array}$ & $\begin{array}{l}\text { Revista Brasileira de Educação } \\
\text { Médica }\end{array}$ & $\begin{array}{l}\text { Transversal de } \\
\text { prevalência }\end{array}$ & BAI \\
\hline $\begin{array}{l}\text { Trindade Jr SCT et } \\
a^{\beta 0}\end{array}$ & $\begin{array}{l}\text { Revista Brasileira de Educação } \\
\text { Médica }\end{array}$ & Transversal quantitativo & MINI \\
\hline
\end{tabular}

FI: fator de impacto; MINI: Minientrevista neuropsiquiátrica internacional; OASIS: Escala Geral de Gravidade e Imparidade da Ansiedade; LSAS-SR: Escala de ansiedade social de Liebowitz; CHQ12: Questionário de Saúde Geral; STAI: Inventário de Ansiedade de Traços do Estado; DSQ-40: Questionário de estilo defensivo; SPIN: Inventário de Fobia Social; PSWQ: Questionário de preocupação da Penn State.

Fonte: dados da pesquisa, 2020.

Ao analisar os artigos selecionados, obteve-se uma amostra total de 6.212 estudantes de medicina brasileiros. Referente às características da amostra citadas no Quadro 2, observa-se maior prevalência do sexo feminino, 2.673 (54,8\%) casos, do que do masculino, 2.673 (43,0\%). Ressalta-se ainda que apenas 1 (5\%) artigo não dispôs a informação sobre o sexo dos participantes. Além disso, acerca do ano da faculdade, 11 (55\%) estudos analisaram os estudantes entre o primeiro e o sexto ano.

Quadro 2. Características da amostra dos estudos incluídos na revisão.

\begin{tabular}{|c|c|c|c|c|c|c|}
\hline Artigo & $\begin{array}{l}\text { Amost } \\
\text { ra (N) }\end{array}$ & $\begin{array}{l}\text { Faixa etária } \\
\text { (a =anos) }\end{array}$ & $\begin{array}{l}\text { Sexo } \\
\text { Masc (\%) } \\
\text { Fem (\%) }\end{array}$ & $\begin{array}{l}\text { Estado } \\
\text { civil } \\
\text { Solt (\%) } \\
\text { Cas (\%) } \\
\end{array}$ & $\begin{array}{l}\text { Ano da } \\
\text { faculdade }\end{array}$ & Outros \\
\hline Bassols AMS et a/ ${ }^{11}$ & 355 & - & $\begin{array}{l}135(38) \\
220(62)\end{array}$ & - & $11^{\circ}$ e $6^{\circ}$ ano & - \\
\hline $\begin{array}{l}\text { Bermudez MB et al } \\
12\end{array}$ & 62 & Média 23,43a & $\begin{array}{l}25(41,6) \\
37(61,7) \\
\end{array}$ & $\begin{array}{l}61(98,4) \\
1(1,6) \\
\end{array}$ & $1^{\circ}$ ao $6^{\circ}$ ano & $\begin{array}{l}\text { Etnia Branco: } 49 \\
\text { Outros: } 13\end{array}$ \\
\hline Borges GBM et al ${ }^{13}$ & 140 & $\begin{array}{l}\text { Entre } 19 \text { e } \\
26 a\end{array}$ & $\begin{array}{l}66(47,1) \\
74(52,9)\end{array}$ & $\begin{array}{l}\text { Solteiro: } \\
86 \\
\text { Estável: } \\
53\end{array}$ & $\begin{array}{ll}1^{\circ}: 41 & 2^{\circ}: \\
43 & \\
4^{\circ}: 33 & 6^{\circ}: \\
22 & \end{array}$ & - \\
\hline Costa DS et a/ ${ }^{14}$ & 279 & Média 22,9a & $\begin{array}{c}139(49,8) \\
137(49,1) \\
\end{array}$ & $\begin{array}{l}273(97,8) \\
6(2,2)\end{array}$ & $1{ }^{\circ}$ ao $6^{\circ}$ ano & - \\
\hline Damiano RF et al ${ }^{15}$ & 431 & Média 21,72a & $\begin{array}{l}193(44,7) \\
238(55,3)\end{array}$ & - & $\begin{array}{l}\text { 10: } 173 \\
\text { 20: } 161 \\
97\end{array}$ & $\begin{array}{l}\text { Empregado } \\
\text { Sim: } 7 \text { Não: } 424\end{array}$ \\
\hline
\end{tabular}




\begin{tabular}{|c|c|c|c|c|c|c|}
\hline Del-Bem CM et al ${ }^{16}$ & 150 & Mediana 56a & $\begin{array}{l}92(38,7) \\
58(61,3) \\
\end{array}$ & - & $1^{\circ}$ ao $6^{\circ}$ ano & $\begin{array}{l}\text { Etnia Branco: } \\
116 \text { Outros: } 34\end{array}$ \\
\hline $\begin{array}{l}\text { Gonçalves JRL et } \\
\text { a/17 }\end{array}$ & 129 & Média 23a & - & - & $1^{\circ}$ ao $6^{\circ}$ ano & - \\
\hline Lucchetti G et al ${ }^{18}$ & 138 & Média 20,65a & $\begin{array}{l}47(34) \\
91(66) \\
\end{array}$ & - & $1^{\circ}$ e $2^{\circ}$ ano & - \\
\hline Machado L et al ${ }^{19}$ & 417 & Média 22a & $\begin{array}{l}208(49,9) \\
209(50,1)\end{array}$ & $\begin{array}{l}404(4,1) \\
17(96,9) \\
\end{array}$ & $1^{\circ}$ ao $4^{\circ}$ ano & - \\
\hline Maia JMC et al ${ }^{20}$ & 129 & Média 23,06aㅗ & $\begin{array}{l}30(23,2) \\
99(76,8)\end{array}$ & - & $1^{\circ}$ ao $6^{\circ}$ ano & - \\
\hline Mayer FB et $a l^{21}$ & 1.350 & Média 22,8aㅗ & $\begin{array}{l}714(52,9) \\
636(47,1) \\
\end{array}$ & - & $1^{\circ}$ ao $6^{\circ}$ ano & - \\
\hline $\begin{array}{l}\text { Moromizato MS et } \\
a^{R^{2}}\end{array}$ & 169 & Mediana 21a & $\begin{array}{l}62(36,7) \\
107(63,3) \\
\end{array}$ & - & $1^{\circ}$ ao $5^{\circ}$ ano & - \\
\hline Motta ICM et $a^{23}$ & 129 & $\begin{array}{l}\text { Entre } 18 \text { e } \\
32 a\end{array}$ & $\begin{array}{l}65(50,4) \\
64(49,6)\end{array}$ & $\begin{array}{l}126(97,7) \\
3(2,3)\end{array}$ & $\begin{array}{l}1^{\circ} \text { e } 2^{\circ}: 62 \\
3^{\circ} \text { e } 4^{\circ}: 45 \\
5^{\circ} \text { e } 6^{\circ}: 22\end{array}$ & - \\
\hline $\begin{array}{l}\text { Moutinho IVD et al } \\
24\end{array}$ & 312 & Média 21 a & $\begin{array}{l}112(36) \\
200(64)\end{array}$ & - & $\begin{array}{ll}10: 93 & 2 \stackrel{\circ}{\circ}: \\
88 & \\
3^{\circ}: 75 & 4 \stackrel{\circ}{*}: \\
56 & \\
\end{array}$ & 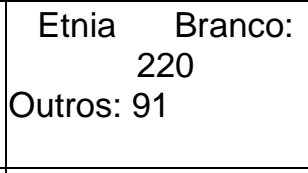 \\
\hline Nogueira EG et al ${ }^{25}$ & 140 & Média 24,3a & $\begin{array}{l}41(29,3) \\
99(70,7)\end{array}$ & $\begin{array}{l}136(97,1) \\
4(2,9)\end{array}$ & $\begin{array}{l}9:: 27 \\
10:: 31 \\
11^{\circ}: 37 \\
45\end{array}$ & $\begin{array}{l}\text { Empregado } \\
\text { Sim: } 52 \text { Não: } \\
88\end{array}$ \\
\hline Regis JMO et al 26 & 479 & Média 22,5a & $\begin{array}{l}198(41,4) \\
281(58,6) \\
\end{array}$ & $\begin{array}{l}474(98,9) \\
4(0,8)\end{array}$ & $1^{\circ}$ ao $6^{\circ}$ ano & - \\
\hline Ribeiro CF et al ${ }^{27}$ & 355 & Média 23,06a & $\begin{array}{l}135(38) \\
220(62) \\
\end{array}$ & - & $1^{0}$ ao $6^{\circ}$ ano & - \\
\hline $\begin{array}{l}\text { Rodrigues MDS et } \\
\text { al }{ }^{28}\end{array}$ & 431 & Média 21,4 a & $\begin{array}{l}165(38,3) \\
266(61,7) \\
\end{array}$ & $\begin{array}{c}413(95,8) \\
18(4,2) \\
\end{array}$ & $1^{\circ}$ ao $4^{\circ}$ ano & - \\
\hline $\begin{array}{l}\text { Sacramento BO et } \\
\text { al }^{29}\end{array}$ & 458 & Mediana 22a & $\begin{array}{l}169(36,9) \\
289(63,1) \\
\end{array}$ & - & $1^{\circ}$ ao $6^{\circ}$ ano & $\begin{array}{l}\text { Etnia Branco: } \\
260 \text { Outros: } 169\end{array}$ \\
\hline $\begin{array}{l}\text { Trindade Jr SCT et } \\
\text { al }{ }^{30}\end{array}$ & 159 & $\begin{array}{l}<18: 18 \\
>23: 42\end{array}$ & $\begin{array}{l}78(49,1) \\
81(50,9) \\
\end{array}$ & $\begin{array}{l}150(94,3) \\
9(5,7)\end{array}$ & $1^{\circ}$ ao $5^{\circ}$ ano & - \\
\hline
\end{tabular}

Masc $=$ masculino; Fem $=$ feminino; Solt $=$ solteiro; Cas $=$ casado

Fonte: dados da pesquisa, 2021.

Através do quadro 3 é possível avaliar a prevalência da ansiedade nessa revisão sistemática, apesar de haver limitações, dado que 7 (35\%) artigos não mencionaram ela. No total, $1.742(28 \%)$ estudantes de medicina apresentaram ansiedade, sendo que $50 \%(n=10)$ dos estudos relataram que o sexo feminino estava relacionado com maiores índices de ansiedade, sendo considerado, por alguns, como um fator de risco. Outros fatores associados a isso foi realização de terapia psicológica ou psiquiátrica, distúrbios no sono, uso de drogas e outros. 
Quadro 3: Níveis de ansiedade e fatores relacionados discutidos nos estudos analisados.

\begin{tabular}{|c|c|c|c|c|c|}
\hline Artigo & $\begin{array}{l}\text { Prevalência } \\
\text { da } \\
\text { ansiedade } \\
(\%)\end{array}$ & $\begin{array}{c}\text { Média de } \\
\text { ansiedade }\end{array}$ & $\begin{array}{l}\text { Sintomas de } \\
\text { ansiedade } \\
\text { (N) }\end{array}$ & $\begin{array}{l}\text { Nível de } \\
\text { ansiedade } \\
\text { (N) }\end{array}$ & $\begin{array}{l}\text { Fatores relacionados } \\
\text { positivamente }\end{array}$ \\
\hline $\begin{array}{l}\text { Bassols AMS } \\
\text { et al }{ }^{11}\end{array}$ & $\begin{array}{l}45(19,5) \\
\text { 10 ano: } 34 \\
\text { 6o ano: } 11\end{array}$ & & - & - & $\begin{array}{l}\text { Ano do curso; uso de álcool, } \\
\text { drogas e medicamentos; } \\
\text { gênero; doença física; } \\
\text { satisfação com o curso. } \\
\text { Relação com mecanismo de } \\
\text { defesa imaturo e neurótico }\end{array}$ \\
\hline $\begin{array}{l}\text { Bermudez MB } \\
\text { et } a l^{12}\end{array}$ & $\begin{array}{l}17(27,4) \\
\text { TAG: } 28 \\
(45,1)\end{array}$ & & - & - & $\begin{array}{l}\text { Realizado intervenção com } \\
\text { UP, com redução do } \\
\text { diagnóstico de TAG para } 20 \\
\text { casos }\end{array}$ \\
\hline $\begin{array}{l}\text { Borges GBM et } \\
\mathrm{a} /{ }^{13}\end{array}$ & - & $\begin{array}{l}\text { Mediana } \\
\text { Início: } 5 \\
\text { Final: } 3\end{array}$ & - & - & $\begin{array}{l}\text { Relação com mecanismo de } \\
\text { defesa imaturo e neurótico }\end{array}$ \\
\hline $\begin{array}{l}\text { Costa DS et al } \\
14\end{array}$ & $\begin{array}{l}\text { Todos (279) } \\
\text { apresentaram } \\
\text { algum } \\
\text { sintoma }\end{array}$ & & $185(66,3)$ & $\begin{array}{l}\text { Leve: } 61 \\
\text { Moderada: } \\
30 \\
\text { Grave: } 3\end{array}$ & $\begin{array}{l}\text { Sexo feminino, atendimento } \\
\text { psicológico prévio, menos } \\
\text { horas de sono e } 1^{\circ} \text { ao } 4^{\circ} \text { ano } \\
\text { do curso }\end{array}$ \\
\hline $\begin{array}{l}\text { Damiano RF et } \\
\text { al }{ }^{15}\end{array}$ & - & 3,98 & - & - & Sexo feminino e estresse \\
\hline $\begin{array}{l}\text { Del-Bem CM et } \\
\text { al }{ }^{16}\end{array}$ & $105(70)$ & - & - & - & $\begin{array}{l}\text { Sem diferença na distribuição } \\
\text { de ansiedade entre currículo } \\
\text { tradicional e reformado do } \\
\text { curso }\end{array}$ \\
\hline $\begin{array}{l}\text { Gonçalves JRL } \\
\text { et } a \Gamma^{7}\end{array}$ & - & 8,04 & - & - & $\begin{array}{l}\text { Religiosidade organizacional } \\
\text { (frequência) foi associada a } \\
\text { menores níveis de ansiedade }\end{array}$ \\
\hline $\begin{array}{l}\text { Lucchetti G et } \\
\text { a/ }{ }^{18}\end{array}$ & - & 4,31 & - & - & $\begin{array}{l}\text { Comparação com alunos dos } \\
\text { Estados Unidos, em que há } \\
\text { mais depressão, ansiedade e } \\
\text { estresse entre os brasileiros }\end{array}$ \\
\hline $\begin{array}{l}\text { Machado L et } \\
\text { al }{ }^{19}\end{array}$ & $19(14,7)$ & & - & - & $\begin{array}{l}\text { Antes de iniciar o curso havia } \\
\text { níveis mais altos de } \\
\text { depressão do que ansiedade, } \\
\text { isso foi revertido durante o } \\
\text { curso }\end{array}$ \\
\hline$\underset{20}{\text { Maia JMC et al }}$ & - & \begin{tabular}{|l}
41,3 \\
Mín: 23 \\
Máx: 66
\end{tabular} & - & - & $\begin{array}{l}\text { Idade (mais jovens), sexo } \\
\text { feminino e outros }\end{array}$ \\
\hline${ }_{21}^{\text {Mayer FB et al }}$ & $\begin{array}{l}149 \text { (11\%) } \\
1^{\circ} \text { e } 2^{\circ} \text { ㅇa: } \\
56 \\
3^{\circ} \text { e e } 4^{\circ} \text { ano: } \\
62 \\
5^{\circ} \text { e } 6^{\circ} \text { ano: } \\
29\end{array}$ & - & $\begin{array}{l}\text { Traço de } \\
\text { ansiedade } \\
\text { Moderado: } 675 \\
\text { Alto: } 480\end{array}$ & $\begin{array}{l}\text { Estado de } \\
\text { ansiedade } \\
\text { Moderado: } \\
693 \text { Alto: } \\
410\end{array}$ & $\begin{array}{l}\text { Sexo feminino, residir em } \\
\text { capital e outros } \\
\text { Sem relação com anos do } \\
\text { curso }\end{array}$ \\
\hline $\begin{array}{l}\text { Moromizato MS } \\
\text { et aR2 }\end{array}$ & - & & - & - & $\begin{array}{l}\text { Efeito prejudicial do uso da } \\
\text { web, interferência no no } \\
\text { rendimento acadêmico e no } \\
\text { sono pelo uso } \\
\text { Sem relação com tempo gasto } \\
\text { na internet }\end{array}$ \\
\hline
\end{tabular}




\begin{tabular}{|c|c|c|c|c|c|}
\hline $\begin{array}{l}\text { Motta ICM et } \\
a^{R^{3}}\end{array}$ & $\begin{array}{l}\text { TAG } 51 \\
(39,5)\end{array}$ & & - & - & $\begin{array}{l}\text { Relação com bullying, com 5o } \\
\text { e 6ano do curso e outros } \\
\text { Há menos alunos com TAG e } \\
\text { que sofrem de depressão e } \\
\text { que mudaram de residência e } \\
\text { outros }\end{array}$ \\
\hline $\begin{array}{l}\text { Moutinho IVD } \\
\text { et al } 24\end{array}$ & $94(47,1)$ & 3,5 & - & - & $\begin{array}{l}\text { Sexo feminino, anos iniciais } \\
\text { do curso, baixa renda, outros }\end{array}$ \\
\hline $\begin{array}{l}\text { Nogueira EG et } \\
a l^{25}\end{array}$ & $66(47,1)$ & & - & $\begin{array}{l}\text { Leve: } 36 \\
\text { Moderada: } \\
21 \\
\text { Severa: } 9\end{array}$ & $\begin{array}{l}\text { Sexo feminino, insônia, } \\
\text { terapia psicológica ou } \\
\text { psiquiátrica, outros. Menor } \\
\text { níveis nos com rendimento } \\
\text { acadêmico satisfeito }\end{array}$ \\
\hline $\begin{array}{l}\text { Regis JMO et } \\
\text { al }{ }^{26}\end{array}$ & - & & $\begin{array}{l}\text { TAS sintomas } \\
\text { em } 174\end{array}$ & $\mid-$ & $\begin{array}{l}\text { Pensamento de abandonar } \\
\text { curso, sintomas depressivos, } \\
\text { terapia } \\
\text { psicológica/psiquiátrica, } \\
\text { outros. }\end{array}$ \\
\hline$\underset{27}{\text { Ribeiro CF et al }}$ & $\begin{array}{l}149 \text { (42) } \\
1^{\circ} \text { e e } 2^{\circ} \text { ano: } \\
56 \\
3^{\circ} \text { e } 4^{\circ} \text { ano: } \\
62 \\
5^{\circ} \text { e } 6^{\circ} \text { ano: } \\
29\end{array}$ & & - & - & $\begin{array}{l}\text { Sexo feminino, sentir-se } \\
\text { sozinho, ter histórico de } \\
\text { tratamento psiquiátrico/ } \\
\text { psicológico e sentir-se } \\
\text { moralmente prejudicado }\end{array}$ \\
\hline $\begin{array}{l}\text { Rodrigues } \\
\text { MDS et al } 28\end{array}$ & $258(59,9)$ & & $\begin{array}{l}258 \\
\text { TAS: } 255\end{array}$ & $\begin{array}{l}\text { Leve: } 115 \\
\text { Moderado: } \\
42 \\
\text { Grave: } 19\end{array}$ & 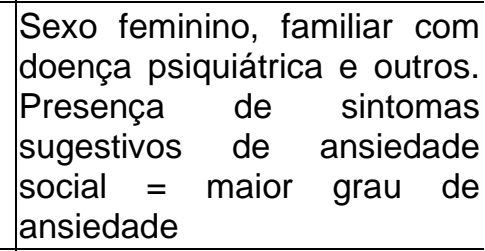 \\
\hline $\begin{array}{l}\text { Sacramento } \\
\text { BO et al }{ }^{29}\end{array}$ & $430(93,9)$ & & 289 (63\%) & $\begin{array}{l}\text { Leve: } 91 \\
\text { Moderada: } \\
39 \\
\text { Grave: } 11\end{array}$ & $\begin{array}{l}\text { Sexo feminino, idade }(<22 \\
\text { anos) e orientação sexual } \\
\text { (homo/ bissexual). Sem } \\
\text { relação com ano do curso }\end{array}$ \\
\hline $\begin{array}{l}\text { Trindade } \mathrm{Jr} \\
\text { SCT et al }{ }^{30}\end{array}$ & $\begin{array}{l}\text { TAG: } \\
\text { 52(32,7) } \\
\text { 10 ano: } 18 \\
\text { 3o ano: } 18 \\
\text { 5o ano: } 16\end{array}$ & - & - & - & $\begin{array}{l}\text { Relação com risco de suicídio, } \\
50 \% \text { dos alunos com esse } \\
\text { risco tinham TAG associado, } \\
\text { enquanto } 25,2 \% \text { dos sem } \\
\text { risco de suicídio tinham TAG }\end{array}$ \\
\hline
\end{tabular}

Mín: mínimo; Máx: máximo; TAG: Transtorno de Ansiedade Generalizada; TAS: Transtorno de Ansiedade Social; UP Protocolo Unificado para Tratamento Transdiagnóstico de Transtornos Emocionais.

Fonte: dados da pesquisa, 2021.

A partir do Quadro 3, é possível analisar os níveis de ansiedade presente nos estudantes de medicina, apesar de que apenas $4(20 \%)$ estudos disponibilizaram dados acerca disso, em que $303(63,5 \%)$ apresentaram nível leve, $132(27,7 \%)$ moderado e $42(8,8 \%)$ grave de ansiedade. Observa-se ainda que os autores Bassols AMS et al11 e Borges GBM et al13 associaram os mecanismos de defesa imaturo e neurótico com a ansiedade.

É válido salientar que houve discrepâncias entre os estudos analisados no que 
diz respeito a relação entre ansiedade e o ano do curso de medicina, em alguns artigos houve associação positiva entre essas variáveis, como em Costa DS et al14 e Moutinho IVD et al24 em que a ansiedade estava relacionada com os anos iniciais do curso, enquanto que em Motta ICM et al23 isso foi verificado nos anos finais (5 e $6^{\circ}$ ano). Ademais, os estudos de Mayer FB et al21 e de Sacramento BO et al29 não observaram relação entre a duração do curso e a ansiedade.

\section{DISCUSSÃO}

Durante a formação profissional, os estudantes da área da saúde acabam apresentando uma tendência maior a manifestar sintomas de ansiedade. Tais graduandos enfrentam vários desafios desde os primeiros anos do curso, englobando desde a mudança radical no cotidiano e estudo até mudança da qualidade de vida e na saúde mental do indivíduo ${ }^{31}$. Isso muitas vezes se deve ao fato de que recai aos médicos a maior parte da expectativa de cura dos pacientes. Dessa maneira, na tentativa de alcançar tal expectativa, o estudante vive envolvido em cargas horárias extremas, não somente na universidade, mas também fora do período letivo ${ }^{32}$.

Associado a isso, têm-se o fato de que as escolas médicas são conhecidas por apresentarem um ambiente de competitividade, com extensas cargas horárias, inúmeras atividades curriculares e extracurriculares, auto cobrança e conteúdos complexos para estudo. Assim, quando associado ao processo de adaptação do estudante, aumenta as chances de se desenvolver transtornos psiquiátricos, com destaque para o de ansiedade ${ }^{33}$.

Diversos métodos de avaliação da ansiedade podem ser utilizados, com destaque para o Inventário de Ansiedade de Beck (BAI), que foi o mais prevalente no presente estudo (30\%). O BAI é composto de 21 itens, sendo que em uma escala de quatro pontos, o indivíduo deve informar o nível de gravidade do sintoma. O instrumento demonstrou-se adequado para utilização não somente em pacientes psiquiátricos, mas também para a população não clínica, como por exemplo, universitários, bombeiros, adolescentes, idosos, funcionários de hospital e de outras empresas, e também para clínica médica ${ }^{34,35}$.

Quanto aos fatores de risco associados, é notória a prevalência de sintomas de ansiedade em mulheres, uma vez que a pesquisa em questão 
observou que tal sexo era responsável por 54,8\% casos. Evidências epidemiológicas têm sugerido que as mulheres possuem uma probabilidade duas vezes maior de preencherem os critérios para Transtorno de Ansiedade Generalizada ao longo da vida. Essas diferenças de gêneros também se tornam evidentes no que diz respeito à apresentação clínica, bem como nas características desses transtornos. Quando comparadas aos homens, mulheres que apresentam transtornos de ansiedade referem maior gravidade dos sintomas e apresentam uma ou mais comorbidades psiquiátricas, com mais frequência ${ }^{36}$.

Diversos outros fatores de risco podem ser apontados em estudantes de medicina. Um fator importante encontrado é a procedência de municípios distantes da universidade. Os estudantes, principalmente aqueles que se afastam da família devido à distância da localização da universidade, tornam-se mais expostos a distúrbios psicológicos. Além disso, a literatura descreve que o fato de o discente dispor de pessoas próximas, com quem possa compartilhar sentimentos, é um elemento importante para impedir o desenvolvimento de processos de estresse e Burnout ${ }^{37,38}$.

O uso de drogas lícitas e ilícitas também pôde ser observado. Segundo um estudo realizado na Faculdade de Medicina da Universidade Federal da Bahia sobre as drogas mais comuns entre os estudantes, foi observado que $56,1 \%$ dos alunos relataram uso de álcool ${ }^{39}$. Quanto aos ansiolíticos $(13,1 \%)$ e as anfetaminas $(10,1 \%)$, esses medicamentos apresentam alto potencial para abuso e são também os mais consumidos. Segundo uma pesquisa realizada na Faculdade de Medicina de São José do Rio Preto, o extenso conteúdo curricular a ser estudado é o que justifica o uso de tais medicamentos, com o intuito de melhorar a atenção e/ou se manter acordados durante mais tempo ${ }^{40}$.

É válido ressaltar, também, o desgaste do curso como um dos principais fatores para o aumento da disseminação dos transtornos mentais entre esses estudantes. O cenário atual tem demostrado tal efeito nocivo na vida do discente de medicina, uma vez que um estudo realizado na Universidade do Estado do Rio Grande do Norte demonstrou que cada vez mais os alunos dispõem de pouco tempo para lazer, refletindo na sensação de sobrecarga decorrente das atividades curriculares e complementares. Apesar disso, muitos se envolvem nestas práticas, buscando melhorar o currículo profissional e adquirir novas habilidades 
técnicas. Tal fato reduz ainda mais a qualidade de vida do acadêmico, repercutindo diretamente nas questões emocionais do indivíduo ${ }^{32,40}$.

Em relação aos níveis de ansiedade nos estudantes de medicina observados, foi demonstrada maior prevalência no nível leve $(63,5 \%)$, seguido de $27,7 \%$ para o moderado e $8,8 \%$ para o grave. No entanto, outras pesquisas demonstram níveis mais elevados de ansiedade, como a realizada na Faculdade de Medicina do ABC paulista, a qual demonstrou que 30,9\% dos graduandos de Medicina apresentaram traços de ansiedade alta, e os demais 69,1\%, ansiedade moderada. Isto deve-se ao fato que uma comparação fidedigna se torna difícil, uma vez que há a utilização de diferentes escalas nos estudos ${ }^{6,38}$.

No presente estudo, observou-se que alguns artigos apontavam maior prevalência da ansiedade nos anos iniciais do curso, enquanto outros afirmavam que há maior prevalência nos anos finais. Sabe-se que o curso de medicina apresenta inúmeros cenários, desde o início da formação acadêmica, que podem desencadear estímulos excessivos de ansiedade, como apresentação de trabalhos, seminários e provas orais ${ }^{42}$.

Entretanto, os dois últimos anos da faculdade - denominados de internato - podem ser um momento que repercute diretamente na ansiedade do aluno, uma vez que os discentes passam a colocar em prática todo conhecimento visto na teoria ao longo dos 4 anos inicias. Sendo assim, acabam por assumir uma postura mais ativa no que diz respeito a tomar condutas, decisões e exercer em sua totalidade a relação médico-paciente ${ }^{43}$.

\section{CONCLUSÃO}

No presente estudo, verificou-se que a maioria dos estudantes de medicina, possuem uma tendência maior a desenvolver sintomas de ansiedade ao decorrer de sua formação profissional, seja nos anos iniciais do curso como também nos dois últimos anos, denominados de internato. Quando comparados aos aspectos sociodemográficos, foi observado que os níveis de ansiedade foram maiores no sexo feminino. Outros fatores associados a isso foi realização de terapia psicológica ou psiquiátrica, distúrbios no sono, uso de drogas e outros.

Os estudantes de Medicina possuem uma tendência maior a desenvolver sintomas de ansiedade ao decorrer de sua formação profissional, pois lidam com 
um curso que exige muito tanto física quanto emocionalmente, principalmente por trabalhar com as dores e os sentimentos de uma pessoa. Os desafios vão desde os primeiros anos, onde é notória a mudança não só na quantidade de estudo, por ser um curso integral, mas também na qualidade de vida e na saúde mental do aluno. Dessa maneira, na tentativa de alcançar a expectativa de cura dos pacientes e por causa dessa grande exigência que recai aos médicos, muitos desses estudantes tendem a desenvolver diversos agravos à saúde mental, dentre estes, a ansiedade. 


\section{REFERÊNCIAS}

1. Dicio, Dicionário Online de Português, definições e significados de mais de 400 mil palavras. Todas as palavras de A a Z. Ansiedade. dicio.com.br, 2021. Disponível em: https://www.dicio.com.br/ansiedade/

2. Sadock BJ, Sadock VA, editors. Kaplan and Sadock's Comprehensive Textbook of Psychiatry. 7th ed. Baltimore: Lippincott Williams \& Wilkins; 2000, p. 2770-81.

3. Leite CD, Silva AA, Angelo LF, Rubio K, Melo GF. Representações de ansiedade e medo de atletas universitários. Rev. Brasileira de Psicologia do Esporte, 2016, 6 (1): 36-46.

4. Andrade LHSG, Gorenstein C. Aspectos gerais das escalas de avaliação de ansiedade. Rev Psiq Clin, 1998; 25 (6):285-290.

5. Caíres MC, Shinohara H. Transtornos de ansiedade na criança: um olhar nas comunidades. Revista Brasileira de Terapias Cognitivas, 2010; 6(1), 62-84.

6. Baldassin S, Martins LC, Andrade AG. Traços de ansiedade entre estudantes de medicina. Arq Med ABC 2006; 31 (1): 27-31.

7. Zonta R, Robles ACC, Grosseman S. Estratégias de enfrentamento do estresse desenvolvidas por estudantes de medicina. Rev Bras Educ Med. 2006;30(3):147-53.

8. Quek TT, Tam WW, Tran BX, Zhang M, Zhang Z, Ho CS, et al. The Global Prevalence of Anxiety Among Medical Students: A Meta-Analysis. Int J Environ Res Public Health. 2019;16(15):2735.

9. Margis R. Comorbidade no transtorno de estresse pós-traumático: regra ou exceção?. Rev Bras Psiquiatr. 2003; 25 (supl I): 17-20.

10. Landis JR, Koch GG. The measurement of observer agreement for categorical data. Biometrics, 1977; 33(1):159-174. 
11. Bassols AMS, Guimarães GC, Ogliari CKM, Santos SM, Carneiro BB, Hirakata VN, et al. How do medical students defend themselves against anxiety?. Rev Bras Psicoterapia. 2017; 19(1):31-42.

12. Bermudez MB, Constanzi M, Macedo MJA, Tatton-Ramos T, Xavier ACM, Ferrão YA, et al. Improved quality of life and reduced depressive symptoms in medical students after a single-session intervention. Braz. J. Psychiatr. 2020; $42(2): 145-152$.

13. Borges GBM, Eidt I, Zilli LN, Michels AMMP, Diaz AP. Defense mechanisms and quality of life of medical students according to graduation phase. Trends Psychiatry Psychother. 2020; 42(1): 74-81.

14. Costa DS, Medeiros NSB, Cordeiro RA, Frutuoso ES, Lopes JM, Moreiro SNT. Sintomas de Depressão, Ansiedade e Estresse em Estudantes de Medicina e Estratégias Institucionais de Enfrentamento. Rev Bras Educ Med. 2020; 44 (1):e040.

15. Damiano RF, Oliveira IN, Ezequiel OS, Lucchetti AL, Lucchetti G. The root of the problem: identifying major sources of stress in Brazilian medical students and developing the Medical Student Stress Factor Scale. Braz. J. Psychiatr. $2021 ; 43$ (1): 35-42.

16. Del-Bem CM, Shuhama R, Costa MJ, Troncon LEA. Effect of changes to the formal curriculum on medical students' motivation towards learning: a prospective cohort study. Sao Paulo Med J. 2019; 137(2):112-8.

17. Gonçalves JRL, Jorge AP, Zanetti GC, Amaro EA, Tótoli RT, Lucchetti G. Religiousness is associated with lower levels of anxiety, but not depression, in medical and nursing students. Rev Assoc Med Bras. 2018; 64(6):537-542

18. Lucchetti G, Damiano RF, DiLalla LF, Lucchetti ALG, Moutinho ILD, da Silva Ezequiel O, et al. Cross-cultural Differences in Mental Health, Quality of Life, Empathy, and Burnout between US and Brazilian Medical Students. Acad Psychiatry. 2018;42(1):62-67. 
19. Machado L, Souza CTN, Nunes RO, Santana CN, Araujo CF, Cantilino A. Subjective well-being, religiosity and anxiety: a cross-sectional study applied to a sample of Brazilian medical students. Trends Psychiatry Psychother. 2018;40(3) - 185-192.

20. Maia JMC, Lewis T, Marques Dos Santos N, Picon F, Kadhum M, Farrell SM, et al. Stressors, psychological distress, and mental health problems amongst Brazilian medical students. Int Rev Psychiatry. 2019;31(7-8):603-607.

21. Mayer FB, Santos IS, Silveira PS, Lopes MHI, de Souza AR, Campos EP, et al. Factors associated to depression and anxiety in medical students: a multicenter study. BMC Med Educ. 2016;16(1):282.

22. Moromizato MS, Ferreira DBB, Souza LSM, Leite RF, Macedo FN, Pimentel D. O Uso de Internet e Redes Sociais e a Relação com Indícios de Ansiedade e Depressão em Estudantes de Medicina. Rev Bras Educ Med. 2017; 41 (4):497504.

23. Motta ICM, Soares RCM, Belmonte TSA. Uma Investigação sobre Disfunções Familiares em Estudantes de Medicina. Rev Bras Educ Med. 2019; 43 (1):4756.

24. Moutinho IVD, Lucchetti ALG, Ezequiel ODS, Lucchetti G. Mental health and quality of life of Brazilian medical students: Incidence, prevalence, and associated factors within two years of follow-up. Psychiatry Res. 2019;274:306312.

25. Nogueira EG, Matos NC, Machado JN, Araújo LB, Silva AMTC, Almeida RJ. Avaliação dos níveis de ansiedade e seus fatores associados em estudantes internos de Medicina. Rev Bras Educ Med. 2021; 45 (1):e017.

26. Regis JMO, Ramos-Cerqueira ATA, Lima MCP, Torres AR. Social anxiety symptoms and body image dissatisfaction in medical students: prevalence and correlates. J Bras Psiquiatr. 2018;67(2):65-73 
27. Ribeiro CF, Lemos CMC, Alt NN, Marins RLT, Corbiceiro WCH, Nascimento MI. Prevalence of and Factors Associated with Depression and Anxiety in Brazilian Medical Students. Rev Bras Educ Med. 2020; 44 (1):e021.

28. Rodrigues MDS, Rocha PBC, Araripe PF, Rocha HAL, Sanders LLO, Kubrusly M. Transtorno de Ansiedade Social no Contexto da Aprendizagem Baseada em Problemas. Rev Bras Educ Med. 2019; 43 (1):65-71.

29. Sacramento BO, Anjos TL, Barbosa AGL, Tavares CF, Dias JP. Symptoms of anxiety and depression among medical students: study of prevalence and associated factors. Rev Bras Educ Med. $2021 ; 45$ (1):e021.

30. Trindade Júnior SC, Sousa LFF, Carreira LB. Generalized anxiety disorder and prevalence of suicide risk among medical students. Rev Bras Educ Med. 2021; 45 (2):e061.

31. Aquino TAA. Atitudes e intenções de cometer suicídio: Seus correlatos existenciais e normativos. Unpublished doctoral dissertation, Federal University of Paraíba, João Pessoa, Brazil, 2009.

32. Neponuceno HJ, Souza BDM, Neves NMBC. Common mental disorders among medical students. Rev. bioét. (Impr.). 2019; 27 (3): 465-70.

33. Adewuya AO, Ola BA, Aloba OO, Mapayi BM, Oginni OO. Depression amongst Nigerian university students. Social psychiatry and psychiatric epidemiology. 2006; 41(8): 674-678.

34. Baptista MN, Carneiro AM. Validade da escala de depressão: relação com ansiedade e stress laboral. Campinas; Estudos de Psicologia 2011; 28(3): 345352.

35. Cunha JA. Manual da versão em português das escalas Beck. São Paulo: Casa do Psicólogo, 2001.

36. Kinrys G, Wygant LE. Transtornos de ansiedade em mulheres: gênero influência o tratamento?. Rev Psiquiatr, 2005; 27 (supl II):S43-50. 
37. Benevides-Pereira AMT. O processo de adoecer pelo trabalho. São Paulo: Casa do Psicólogo; 2002: 105-132

38. Vasconcelos TC, Dias BRT, Andrade LR, Melo GF, Barbosa L, Souza E. Prevalência de Sintomas de Ansiedade e Depressão em Estudantes de Medicina. Rev Bras Educ Med. 2015; 39 (1):135-142.

39. Almeida AM, Godinho TM, Bitencourt GV. Common mental disorders among medical students. J Bras Psiquiatr. 2007. 56(4): 245-251.

40. Pinton FA, Boskovitz EP, Cabrera EMS. Uso de drogas entre os estudantes de medicina da faculdade de medicina de São José do Rio Preto. Arq Cienc Saúde. 2002; 12(2): 91-96.

41. Cardoso Filho FAB, Magalhães JF, Silva KML, Pereira ISSD. Perfil do estudante de medicina da Universidade do Estado do Rio Grande do Norte. Rev Bras Educ Méd. 2015;39(1):32-40.

42. Oliveira MA, Duarte ÂMM. Controle de respostas de ansiedade em universitários em situações de exposições orais. Revista Brasileira de Terapia Comportamental e Cognitiva. 2004; 6(2): 183-199.

43. Alves TCDTF. Depressão e ansiedade entre estudantes da área de saúde. Revista de Medicina; 2004; 93(3): 101-105. 